\title{
EXECUTIVE CONTROL ABILITIES AND SELF-REGULATION IN SURVIVORS OF CHILDHOOD ACUTE LYMPHOCYTIC LEUKEMIA
}

Amara Gul ${ }^{\otimes}$, Sadia Zafar

\section{ABSTRACT}

OBJECTIVE: To examine executive control and self-regulation abilities in survivors of childhood acute lymphocytic leukemia (ALL).

METHODS: In this cross-sectional study, thirty-five survivors of childhood ALL from Sahukat Khanum Memorial Cancer Hospital, Children Hospital and Jinnah Hospital, Lahore, Pakistan were selected through convenient sampling technique from March 2013 to February 2014. Thirty-five demographically matched healthy children were recruited from local community as controlgroup. Trail Making Test (TMT) and Self-regulation Questionnaire was administered to childhood ALL survivors and healthy children by researchers.

RESULTS: Mean age of ALL survivors and healthy children was 14.25 \pm 2.7 I years and $14.34 \pm 2.98$ years respectively. ALL group showed significant executive control deficit as compared to control group. Mean TMT-part A was $35.25 \pm 2.38$ seconds and $16.14 \pm 2.80$ seconds in ALL group and control group respectively $(\mathrm{p}<0.00 \mathrm{I})$ while mean TMT-part B was $105.62 \pm 2.38$ seconds \& $33.42 \pm 2.30$ seconds in ALL group and control group respectively $(\mathrm{p}<0.00 \mathrm{I})$. In addition, both groups performed significantly different on self-regulation questionnaire. ALL group as compared to control group showed impaired emotional regulation $(18.65 \pm 1.10$ seconds vs. $5.57 \pm 0.85$ seconds respectively; $\mathrm{p}<0.00 \mathrm{I})$; behavioral regulation ( $14.97 \pm 0.92$ seconds vs. $4.68 \pm 0.75$ second respectively; $\mathrm{p}<0.00 \mathrm{I})$; and cognitive regulation ( $3.57 \pm 0.85$ seconds vs. $10.60 \pm \mathrm{I} .55$ seconds respectively; $\mathrm{p}<0.00 \mathrm{I}$ ).

CONCLUSION: Survivors of childhood ALL have marked deficits in social cognition. Treatment protocols might also focus on psychosocial deterioration for better patient care.

KEY WORDS: Executive Function (MeSH); Emotion (MeSH); Trail Making Test $(\mathrm{MeSH})$; Self-Regulation Questionnaire (Non-MeSH); Acute Lymphocytic Leukemia (Non-MeSH); Lymphoproliferative Disorders (MeSH); Precursor BCell Lymphoblastic Leukemia-Lymphoma (MeSH); Precursor T-Cell Lymphoblastic Leukemia-Lymphoma (MeSH); Pediatric Cancer (Non-MeSH); Child (MeSH).

THIS ARTICLE MAY BE CITED AS: Gul A, Zafar S. Executive control abilities and self-regulation in survivors of childhood acute lymphocytic leukemia. Khyber Med Univ J 2018;10(I):3-7.

\section{INTRODUCTION}

ancer is emerging as a major cause of mortality all around world. Pediatric cancer is challenging experience for the child as well as family.' Leukemia is the type of cancer affecting bone marrow and blood. Acute lymphocytic leukemia (ALL) is common type of leukemia and is a result of malignant production of lymphoid precursor cells in bone marrow and blood. $^{2}$ Symptoms of ALL include breathing problems, infections, laziness, fever and blood loss. It is estimated that Pakistani children with ALL have higher burdens of Leukemia which might result in poor prognosis. ${ }^{3}$ Though treatment protocols for ALL has been advanced over the years which increased survival $1^{\otimes}$ The Islamia University of Bahawalpur, Pakistan

Email:amara_psychology@hotmail.com

Date Submitted: February 22, 2017

Date Revised: December 13,2017

Date Accepted: December 30, 2017

rate, yet deficits in several areas of cognition, emotion and behavior have been demonstrated by ALL survivors. ${ }^{4}$ Treatment protocols destroy leukemic cells present in bone marrow, cerebrospinal fluid and organs. Moreover, treatment inhibits disease metastasis in the central nervous system. ${ }^{3}$ Survivors of ALL treated with chemotherapy only showed cognitive deficits in verbal functions, attention, and visual-spatial problem solving. ${ }^{5}$ Neurocognitive impairment was also marked in ALL survivors treated without cranial radiation. ${ }^{6}$ Behavioral and emotional problems have also been observed in childhood malignancies specifically ALL. ${ }^{7}$ It has been suggested that cognitive impairment is associated with difficulties in emotion regulation and deficient coping skills in survivors of ALL. ${ }^{8}$ Mechanism of neurocognitive deficits are associated with chemotherapy agents which are neurotoxic in nature. Drugs administered intravenously or given orally have adverse effects on cognitive, emotional and behavioral functioning, for instance Methotrexate is associated with changes in white matter of the brain and neurocognitive performance, paralysis and seizures of acute nature. ${ }^{9,10}$ Corticosteroids are associated with reduced hippocampal activity and behavioral problems." Vincristine causes peripheral neuropathy. ${ }^{12}$ Standard treatment protocol also includes periodic intrathecal methotrexate and corticosteroids directly injected in to the central nervous system. This therapy has exacerbated neurotoxicity. ${ }^{13}$ In addition, there are certain structural brain changes in ALL survivors which are associated with cognitive deficits and difficulties in emotion regulation such as reduced volumes of the prefrontal cortex and cerebral white matter. ${ }^{14}$ Cerebral hemorrhages are common in ALL survivors cured with cerebral radiation therapy. ${ }^{15}$ Intrathecal methotrexate and 
cranial irradiation are associated with hemosiderin and white matter lesions in ALL survivors. ${ }^{16,17}$ Though previous studies have examined long-term consequences on cognition, emotion and behavior, but there are no studies in literature which have examined cognitive control and self-regulation abilities in ALL survivors. Therefore; the aim of the present study was to assess executive control and self-regulation abilities of ALL survivors. The study examined the question whether ALL survivors cured with systematic chemotherapy excluding cranial radiation show any differential performance on executive function and self-regulation as compared with healthy individuals. Given the treatment related neurotoxic effects and structural brain changes, it was hypothesized that ALL survivors would be deficient in executive control, emotional, behavioral and cognitive regulation in contrast to healthy control subjects.

\section{METHODS}

The study had a cross-sectional research design. Thirty-five children and adolescent survivors of childhood ALL at Jinnah Hospital, Children Hospital and Shaukat Khanum Memorial Cancer Hospital Lahore and thirty-five demographically matched healthy control children participated in the study from March 2013 until February
2014 (Table I). ALL survivors completed treatment protocol at Jinnah Hospital, Children Hospital and Shaukat Khanum Memorial Cancer Hospital, Lahore, Pakistan. Inclusion criterion for ALL survivors was as follows: (i) completed the treatment protocol for standard or high risk childhood ALL (ii) continuous first remission without relapse. Exclusion criterion for ALL group were as follows: (i) history of CNS pathology, bone marrow transplant, other cancer diagnosis, or major medical illness (ii) neurological or psychiatric disorder. Convenient sampling was used. Patients who were willing to participate in the study were included. The inclusion criterion for healthy control children were: (i) no history or present diagnosis of cancer (ii) no known neurological or psychiatric illness.

\section{Measures}

\section{Trail Making Test (TMT)}

$T M T^{18}$ is a neuropsychological instrument to examine executive control abilities. The test consists of two parts. In Part A, subjects are required to connect numbered circles whereas part $B$ required subjects to connect a series of numbered-lettered circles alternating between these sequences. TMT part $A$ reflects visuoperceptual abilities and part B indicates working memory and task switching abilities. TMT was administered to thirty-five childhood survivors of $A L L$ and thirty-five healthy children (participants of present study) by researchers themselves. The time taken by the participants was recorded through a stop watch. Chronbach's alpha $=.80, \mathrm{p}<0.00 \mathrm{I}$ and test-retest reliability after 2 months $(r=.85$, $\mathrm{p}<0.00 \mathrm{I}$ ) were high for sample in the current study. TMT has been used previously to examine visuomotor scanning speed (part A) and executive functions (part B) validated in patients with various types of cancer, for instance acute myelogenous leukemia, ${ }^{19,20}$ patients with cancer in palliative care, ${ }^{21}$ patients with ALL. ${ }^{22}$

\section{Self-Regulation Questionnaire}

Self-regulation questionnaire ${ }^{23}$ measures emotional, cognitive and behavioral control on three separate subscales. It is a I3-item questionnaire. Each item is to be assessed how true is the statement for the subject on response categories ranging from ( $I=$ never true) to ( $4=$ always true). Higher scores indicate deficits on emotional and behavioral subscales whereas lower scores on cognitive regulation subscale show impairment. Emotional regulation scale examines the ability to control anger, intense emotional reaction, and regulation of negative emotions. Cognitive regulation measures the ability to control impulse, focus attention to tasks, thinking and

TABLE I: DEMOGRAPHIC AND CLINICAL CHARACTERISTICS OF ACUTE LYMPHOCYTIC LEUKEMIA SURVIVORS GROUP AND CONTROL GROUP

\begin{tabular}{|c|c|c|c|}
\hline & $\begin{array}{l}\text { Acute Lymphocytic } \\
\text { Leukemia Survivors Group } \\
\qquad(n=35)\end{array}$ & $\begin{array}{l}\text { Control Group } \\
\qquad(n=35)\end{array}$ & \\
\hline $\begin{array}{l}\text { Age (Mean } \pm S D) \\
\text { (range I0-20 years) }\end{array}$ & $|4.25 \pm 2.7|$ Years & $14.34 \pm 2.98$ Years & $t(34)=0.33, p=0.73$ \\
\hline $\begin{array}{l}\text { Gender male/female } \\
\text { (male/female \%) }\end{array}$ & $\begin{array}{c}17 / 18 \\
(48.57 / 51.42 \%)\end{array}$ & $\begin{array}{c}17 / 18 \\
(48.57 / 51.42 \%)\end{array}$ & \\
\hline \multicolumn{4}{|l|}{ Socioeconomic class } \\
\hline High (Frequency/\%) & $11 / 31.42$ & $11 / 31.42$ & \\
\hline Medium (Frequency/\%) & $12 / 34.28$ & $12 / 34.28$ & \\
\hline Low (Frequency/\%) & $12 / 34.28$ & $12 / 34.28$ & \\
\hline $\begin{array}{l}\text { Age (Mean } \pm \text { SD) at time of diagnosis } \\
\text { (range I-I4 years) }\end{array}$ & $6.97 \pm 3.24$ & NA & \\
\hline $\begin{array}{l}\text { Time (Mean } \pm \text { SD) since treatment } \\
\text { completion (range I-I4 years) }\end{array}$ & $7.48 \pm 2.62$ & Nil & \\
\hline
\end{tabular}


EXECUTIVE CONTROL ABILITIES AND SELF-REGULATION IN SURVIVORS OF CHILDHOODACUTE LYMPHOCYTIC LEUKEMIA

TABLE II: DIFFERENCES ON TRAIL MAKING TEST AND SELF-REGULATION QUESTIONNAIRE IN ACUTE LYMPHOCYTIC LEUKEMIA SURVIVORS GROUP AND CONTROL GROUP

\begin{tabular}{|l|c|c|c|c|}
\hline & $\begin{array}{c}\text { Acute Lymphocytic } \\
\text { Leukemia Survivors Group } \\
(\mathrm{n}=35)\end{array}$ & $\begin{array}{c}\text { Control Group } \\
(\mathrm{n}=35)\end{array}$ & & \\
\cline { 2 - 5 } & Mean \pm SD (Seconds) & Mean \pm SD (Seconds) & $\mathbf{t}$ & $\mathrm{p}$-value \\
\hline TMT-part A & $35.25 \pm 2.38$ & $16.14 \pm 2.80$ & $\mathrm{t}(34)=30.82$ & $\mathrm{p}<0.00 \mathrm{I}$ \\
\hline TMT-part B & $105.62 \pm 2.38$ & $33.42 \pm 2.30$ & $\mathrm{t}(34)=117.98$ & $\mathrm{p}<0.00 \mathrm{I}$ \\
\hline ER & $18.65 \pm 1.10$ & $5.57 \pm 0.85$ & $\mathrm{t}(34)=53.67$ & $\mathrm{p}<0.00 \mathrm{I}$ \\
\hline BR & $14.97 \pm 0.92$ & $4.68 \pm 0.75$ & $\mathrm{t}(34)=49.6 \mathrm{I}$ & $\mathrm{p}<0.00 \mathrm{I}$ \\
\hline CR & $3.57 \pm 0.85$ & $10.60 \pm 1.55$ & $\mathrm{t}(34)=22.22$ & $\mathrm{p}<0.00 \mathrm{I}$ \\
\hline
\end{tabular}

$\mathrm{TMT}=$ Trail Making Test; ER=Emotion Regulation; $\mathrm{BR}=$ Behavioral Regulation; $\mathrm{CR}=$ Cognitive Regulation

planning. Behavioral regulation identifies control over hyperactivity and aggression. Self-regulation has been identified as a marker of health in quality of life research. It has been found that this ability is deteriorated in patients with various types of cancer (e.g., breast cancer, colorectal cancer). ${ }^{24}$ Selfregulation questionnaire was administered to the sample of the current study. Psychometric properties for the present sample was high (internal consistency Chronbach's alpha $=.85, \mathrm{p}<0.00 \mathrm{I}$; test-retest reliability after 2 months $r=.89, \mathrm{p}<0.00 \mathrm{I}$ ).

\section{Statistical Analysis}

Demographic and clinical variables were analyzed through descriptive statistics. Group differences on TMT and self-regulation scale were assessed through t-test.

\section{Procedure}

The study was approved by the board of studies of The Islamia University of Bahawalpur, Pakistan. All participants and their parents gave written informed consent. Participants were given instructions to complete TMT as quickly as possible. They were told that the test will measure higher order cognition and they had to complete two parts of TMT. In part A, they had to connect a trail consisting of numbers encircled and in part $B$, they had to complete the trail which alternates between numbers and letters I-A-2-B-3-C, etc. Professional psychologists administered the test and recorded completion time on both parts of TMT in seconds. Then, they were given instructions to complete self-regulation questionnaire. They were told that the questionnaire contains statements which examine your ability to regulate negative emotional and behavioral reactions. After completion of questionnaire, they were debriefed and thanked for their participation.

\section{RESULTS}

Descriptive statistics were computed for demographic and clinical characteristics (Table I) which showed ALL and control groups were matched on gender, age, and socioeconomic status. Mean age of ALL survivors and healthy children was $14.25 \pm 2.7$ I years $14.34 \pm 2.98$ years respectively.

TMT scores were recorded as the time (seconds) taken to perform part $A$ and part $B$ by the ALL and control group. Mean TMT-part A was $35.25 \pm 2.38$ seconds and $16.14 \pm 2.80$ seconds in ALL group and control group respectively $(p<0.00 \mathrm{I})$ and mean TMTpart B was $105.62 \pm 2.38$ seconds \& $33.42 \pm 2.30$ seconds in ALL group and control group respectively $(\mathrm{p}<0.00 \mathrm{I})$.

In addition, both groups performed significantly different on self-regulation questionnaire. ALL group in contrast to control group showed impaired emotional regulation; behavioral regulation and cognitive regulation (Table II).

\section{DISCUSSION}

The present study was conducted to examine executive control and selfregulation in survivors of childhood ALL. There were few important findings: (i) survivors of childhood ALL showed deficits in executive control in contrast with healthy control children.
Executive control deficits extended over part A and part B of TMT. Part A reflected visual attention and part $B$ showed task switching and set-shifting abilities. (ii) ALL survivors showed deficits on emotional regulation, behavioral regulation and cognitive regulation contrary with healthy control children. These results are consistent with previous studies which showed neurological complications in survivors of $A L L^{6,7}$ related with neurotoxic effects of treatment. Executive control and self-regulation are mainly function of prefrontal cortex of the brain. ${ }^{31}$ Deficits in these cognitive areas are consistent with previous findings of reduced volumes in prefrontal cortex and cerebral white matter of the brain, ${ }^{16,17}$ presence of cerebral hemorrhage, hemosiderin and lesions of the white matter in survivors of childhood ALL. ${ }^{18-20}$ In contrast, healthy children showed efficient performance on TMT which reflected intact activity of the neocortical region (i.e., prefrontal cortex). Healthy children demonstrated no deficits in three areas of selfregulation (i.e., cognitive, emotional and behavioral). These results are consistent with previous studies which assessed cognitive impairment in patients with various types of cancer. Patients with Acute Myelogenous Leukemia and Myelodysplastic syndrome experience neurocognitive impairment and poor executive function. ${ }^{24}$ Time taken to perform TMTpart A Mean \pm SD 49.04 232.26 and TMT-part B Mean \pm SD I I 8.85 \pm 84.48 are also consistent with results of the current study. Patients with breast cancer and colorectal cancer performed TMT-part B in Mean = I 32 seconds (SD 
68.2) ${ }^{25}$ Executive functioning has not been examined through TMT in Pakistani population suffering from ALL, therefore; scores are not available for comparison. Similarly, it has been found that patients with cancer exhibit negative coping styles and psychological distress, ${ }^{25}$ but data on self-regulation are missing in existing literature. Thus, findings of the present study are taken as preliminary evidence.

In conclusion, results of the present study suggest deficient executive control and self-regulation abilities in survivors of ALL. Results have implications for management and prevention of cognitive impairment at early stage of ALL. Psychotherapy and cognitive rehabilitation focusing emotion, behavioral and cognitive regulation might be used besides treatment protocol in order to improve skills for daily life functioning in survivors of ALL. Limitation of the study is its' small sample size. Future studies should examine whether executive control and self-regulation can be improved with training in patients with ALL.

\section{REFERENCES}

I. Woodgate RL, Degner FL, Yanofsky RA. Different perspective to approaching cancer symptoms in children. J Pain Symptom Manage 2003 Sep;26(3):800- 17.

2. Mayo Foundation for Medical Education and Research. Diseases and conditions Leukemia. USA: Mayo Clinic, 2016.

3. Yasmeen N, Ashraf S. Childhood Acute lymphoblastic Leukemia: Epidemiology and Clinicopathological features. J Pak Med Assoc 2009 Mar;59(3):I50-3.

4. American Cancer Society. Survival rates for childhood Leukemia. USA: 2016.

5. National Cancer Institute. Childhood Acute Lymphoblastic Leukemia. 2017. USA: US Department of Health and Human Services.

6. Lofstad EG, Reinfjell T, Hestad K, Diseth HT. Cognitive outcome in children and adolescents treated for acute lymphoblastic leukemia with chemotherapy only. Acta Paediatr 2009 Jan;98(I): 180-6. DOI: |0.1 || |/j.|65|-2227.2008.0I055.x.

7. Nazari S, Koupaei MTS, Shafiee A, Kashani ZHG, Bahraminia E, Ansari $M$, et al. Emotional/Behavioral problems in children with Acute Lymphoblastic Leukemia: a case control study. Int J Hematol Oncol Stem Cell Res 20।4;8(2): 14-20.

8. Williams LK, Lamb KE, McCarthy MC. Behavioral side effects of pediatric acute lymphoblastic leukemia treatment: the role of parenting strategies. Pediatr Blood Cancer 20 I 4;6 I (I I):2065-70. DOI: 10.1002/pbc.25164.

9. Campbell KL, Scaduto M, Van Slyke D, Niarhos F, Whitlock AJ, Compas EB. Executive Function, Coping, and Behavior in Survivors of Childhood Acute Lymphocytic Leukemia. J Pediatr Psychol 2009;34(3):31727. DOI: I0.1093/jpepsy/jsn080.

10. Li Y, Vijayanathan V, Gulinello M, Cole PD. Intrathecal methotrexate induces focal cognitive deficits and increases cerebrospinal fluid homocysteine. Pharmacol Biochem Behav 2010 Jun;95(4):428-33. DOI: 10.1016/j.pbb.2010.03.003.

I I. Pound CM, Clark C, Ni A, Athale U, Lewis V, Halton JM. Corticosteroids, behavior, and quality of life in children treated for acute lymphoblastic leukemia; a multicentered trial. J Pediatr Hematol Oncol 2012 Oct;34(7):5 I7-23.

12. Sze Cl, Lin YC, Lin YJ, Hsieh TH, Kuo MY, Lin $\mathrm{CH}$. The role of glucocorticoid receptors in dexamethasone induced apoptosis of neuroprogenitor cells in the hippocampus of rat pups. Mediators Inflamm 2013;2013:628094. DOI: I0.1I55/2013/628094.

13. Jaggi AS, Singh N. Mechanisms in cancer chemotherapeutic drugsinduced peripheral neuropathy. Toxicology 2012 Jan 27;29I(I-3): I 9. DOI: 10.1016/j.tox.20II.10.019.

14. Reddick WE, Shan ZY, Glass JO, Helton S, Xiong $\mathrm{X}, \mathrm{Wu} \mathrm{S}$, et al. Smaller White-Matter Volumes Are Associated with Larger Deficits in
Attention and Learning among Long-Term Survivors of Acute Lymphoblastic Leukemia. Cancer 2006 Feb; I06(4):94I-9.

15. Espy KA, Moore IMK, Kaufmann PM, Kramer JH, Matthay K, Hutter JJ. Chemotherapeutic CNS prophylaxis and neuropsychologic change in children with acute lymphoblastic leukemia: a prospective study. J Pediatr Psychol 200 I Jan-Feb;26(I): I-9.

16. Chan MSM, Roebuckc JD, Yuenb PM, Lib KC, Chan LY. MR Imaging of the brain in patients cured of Acute Lymphoblastic Leukemia-the value of gradient echo Imaging. Am J Neuroradiol 2006 March;27(3):548-52.

17. Laitt RD, Chambers EJ, Goddard PR, Pyhtinen J, Lanning MB. Magnetic resonance imaging and magnetic resonance angiography in long term survivors of acute lymphoblastic leukemia treated with cranial irradiation. Cancer I995 Nov I5;76(10): | 846-52.

18. Reitan RM., Wolfson D. The HalsteadReitan Neuropsycholgical Test Battery: Therapy and clinical interpretation. Tucson, AZ: Neuropsychological Press, 1985.

19. Meyers CA, Albitar M, Estey E. Cognitive impairment, fatigue, and cytokine levels in patients with acute myelogenous leukemia or myelodysplastic syndrome. Cancer 2005 Aug; I 04(4):788-93.

20. Meadows M-E, Chang G, Jones JA, Antin JR, Orav EJ. Predictors of Neuropsychological Change in Patients with Chronic Myelogenous Leukemia and Myelodysplastic Syndrome. Arch Clin Neuropsychol 20I3;28(4):363-74. DOI: 10.1093/arclin/acs |4 |

21. Santos J, Pimenta CA, Kurita GP, Braga PE, Sjøgren P. Validation of the Trail Making Test $B$ for the cognitive assessment of patients with cancer in Palliative Care. Open J Stat $20 । 4 ; 4: 435-45$. D O I: I0.4236/ojs.20|4.46042.

22. Lesnik GP, Ciesielski TK, Hart LB, Benzel CE, Sanders AJ. Evidence for cerebellar-frontal subsystem 
changes in children treated with intrathecal chemotherapy for Leukemia- Enhanced data analysis using an effect size model. Arch Neurol 1998 Dec;55(I2):I56I-8.

23. Novak SP, Clayton RR. The influence of school environment and selfregulation on transitions between stages of cigarette smoking: A multilevel analysis. Healthy Psychol 200।:20(3); 196-207.

24. Büssing A, Girke $M$, Heckmann $C$, Schad F, Ostermann T, Kröz M. Validation of the self-regulation questionnaire as a measure of health in quality of life research. Eur J Med
Res 2009 May |4;| 4(5):223-7. DOI:I0.I |86/2047-783X-|4-5-223.

25. Liu Y, Cao C. The relationship between family history of cancer, coping style and psychological distress. Pak J Med Sci 2014 May;30(3):507-I0. DOI: 10.12669/pjms.303.4634.

\section{AUTHOR'S CONTRIBUTION}

Following authors have made substantial contributions to the manuscript as under:

AG: Concept \& study design; acquisition, analysis \& interpretation of data, drafting the manuscript, final approval of the version to be published

SZ: Acquisition of data, critical review, drafting the manuscript, final approval of the version to be published

Authors agree to be accountable for all aspects of the work in ensuring that questions related to the accuracy or integrity of any part of the work are appropriately investigated and resolved.

This is an Open Access article distributed under the terms of the Creative Commons Attribution-NonCommercial-NoDerivatives 4.0 International License (https://creativecommons.org/licenses/by-nc-nd/4.0/) which permits to reproduce freely in any medium and share the Licensed Material, for NonCommercial purposes only, provided the original work is properly cited.

KMUJ web address: www.kmuj.kmu.edu.pk

Email address: kmuj@kmu.edu.pk 